\title{
Correlation Effects on the Coupled Plasmon Modes of a Double Quantum Well
}

Hill, N. P. R.; Nicholls, J. T.; Linfield, E. H.; Pepper, M.; Ritchie, D. A.; Jones, G. A. C.; Hu, Ben Yu-Kuang; Flensberg, Karsten

Published in:

Physical Review Letters

Link to article, DOI:

10.1103/PhysRevLett.78.2204

Publication date:

1997

Document Version

Publisher's PDF, also known as Version of record

Link back to DTU Orbit

Citation (APA):

Hill, N. P. R., Nicholls, J. T., Linfield, E. H., Pepper, M., Ritchie, D. A., Jones, G. A. C., Hu, B. Y-K., \& Flensberg, K. (1997). Correlation Effects on the Coupled Plasmon Modes of a Double Quantum Well. Physical Review Letters, 78(11), 2204-2207. https://doi.org/10.1103/PhysRevLett.78.2204

\section{General rights}

Copyright and moral rights for the publications made accessible in the public portal are retained by the authors and/or other copyright owners and it is a condition of accessing publications that users recognise and abide by the legal requirements associated with these rights.

- Users may download and print one copy of any publication from the public portal for the purpose of private study or research.

- You may not further distribute the material or use it for any profit-making activity or commercial gain

- You may freely distribute the URL identifying the publication in the public portal 


\title{
Correlation Effects on the Coupled Plasmon Modes of a Double Quantum Well
}

\author{
N.P. R. Hill, J. T. Nicholls, E. H. Linfield, M. Pepper, D. A. Ritchie, and G. A. C. Jones \\ Cavendish Laboratory, University of Cambridge, Madingley Road, Cambridge, CB3 OHE, United Kingdom \\ Ben Yu-Kuang $\mathrm{Hu}$ \\ Mikroelektronik Centret, Danmarks Tekniske Universitet, Bygning 345ø, DK-2800 Lyngby, Denmark \\ Karsten Flensberg \\ Dansk Institut for Fundamental Metrologi, Bygning 307, Anker Engelundsvej 1, DK-2800 Lyngby, Denmark
}

(Received 20 December 1996)

At temperatures comparable to the Fermi temperature, we have measured a plasmon enhanced Coulomb drag in a GaAs/AlGaAs double quantum well electron system. This measurement provides a probe of the many-body corrections to the coupled plasmon modes, and we present a detailed comparison between experiment and theory testing the validity of local field theories. Using a perpendicular magnetic field to raise the magnetoplasmon energy we can induce a crossover to singleparticle Coulomb scattering. [S0031-9007(97)02591-X]

PACS numbers: 73.40.Ty, 71.45.Gm, 72.10.-d

Quantum many-body correlations give rise to novel physics, especially in restricted dimensions where interaction effects are greatly enhanced. For example, twodimensional electron gases (2DEGs) created at a GaAs/ AlGaAs heterojunction have proven to be fertile systems in which correlations reveal themselves in a striking multitude of phenomena. In these systems, the kinetic energy can be quenched to the extent that correlations dominate, giving rise to effects such as the fractional quantum Hall effect and Wigner crystallization. Furthermore, in doublelayer electron systems, it has been shown that correlations lead to interesting phenomena such as new states in the fractional quantum Hall regime. Collective modes in coupled electron gases represent another significant area for the study of many-body effects in reduced dimensions; for example, Neilson et al. [1] showed that the low lying acoustic plasmon mode of the double-layer system is strongly affected by correlations.

In zero magnetic field $(B=0)$ correlation effects have been investigated by both optical [2] and compressibility [3] measurements. Transport measurements, on the other hand, while being a good probe of single-particle behavior, are generally a much less sensitive probe of the correlations. However, one particular type of transport measurement, Coulomb drag in double-layer electron systems, is predicted [4] to be a sensitive probe of the doublelayer plasmon modes. Since the plasmon modes are affected by correlations, this allows the unique possibility for a transport measurement to probe many-body correlations in a two-dimensional system.

In this Letter we present the first transport measurements confirming that coupled plasmons can be probed by Coulomb drag. We compare the experimental data with theoretical results based on the random phase approximation (RPA) with and without local field corrections in the Hubbard approximation [5]. The theory reproduces the data well without any free fitting parameters. However, the data leave some open questions about the applicability of local field corrections at elevated temperatures.

In a drag measurement of two closely spaced, electrically isolated 2DEGs, a drag voltage $V_{\text {drag }}$ is induced in one layer when a current $I_{\text {drive }}$ is passed through the other [6]. Electron-electron interactions transfer momentum from the drive layer (with carrier density $n_{\text {drive }}$ ) to the drag layer (with density $n_{\text {drag }}$ ). The transresistivity is defined as

$$
\rho_{t}=\frac{V_{\text {drag }}}{I_{\text {drive }}} \frac{W}{L},
$$

where $W$ is the width of the Hall bar, $L$ is the distance between voltage probes in the drag layer, and has been calculated [7-9] to be

$$
\begin{aligned}
\rho_{t}= & -\frac{\hbar^{2}}{8 \pi^{2} e^{2} n_{\text {drag }} n_{\text {drive }} k_{B} T} \int_{0}^{\infty} d q \int_{0}^{\infty} d \omega q^{3} \\
& \times\left|\frac{V(q)}{\epsilon(q, \omega)}\right|^{2} \frac{\operatorname{Im} \chi(q, \omega)_{\text {drive }} \operatorname{Im} \chi(q, \omega)_{\mathrm{drag}}}{\sinh \left(\hbar 2 k_{B} T\right) 2},
\end{aligned}
$$

where the static interlayer Coulomb interaction is $V(q)$, $\epsilon(q, \omega)$ is the dielectric function, and the charge density fluctuations in a given layer are characterized by the polarizability $\operatorname{Im} \chi(q, \omega)$. At low temperatures and zero magnetic field, Eq. (2) predicts a $\rho_{t} \sim T^{2}$ temperature dependence, [7] in approximate agreement with experiment [6]. Deviations from $T^{2}$ behavior at low $T$ have been attributed to the exchange of phonons [10]. In a perpendicular magnetic field $B$ the effects of Landau quantization on the drag have been investigated at low temperatures [11-13]; at higher temperatures the transresistivity varies as $\rho_{t} \sim B^{2} T[13]$.

At low temperatures the single-particle excitation (SPE) spectrum for wave vectors much less than the Fermi wave 
vector, $q \ll k_{F}$, is bounded by a line of gradient $v_{F}$ (the Fermi velocity). The plasmon dispersion curves $\omega_{p}(q)$ for a double 2DEG system consist of two branches, both of which lie higher in energy than the SPE spectrum [14-16]. The lower (upper) branch is the acoustic (optic) plasmon, where the charge density oscillations in the two layers are in antiphase (phase). At $T=0$ the real part of the dielectric function is zero, $\operatorname{Re} \epsilon\left(q, \omega_{p}\right)=0$, leading to an "antiscreening" of the interlayer Coulomb interaction for $\omega_{p}(q)$. However, at low $T$ there is no coupling between the SPEs and the plasmons $\left[\operatorname{Im} \chi\left(q, \omega_{p}\right)=0\right]$ and consequently there is no plasmon enhancement of $\rho_{t}$. At temperatures of the order of the Fermi temperature, $T \sim T_{F}$, the SPE spectrum is sufficiently broadened to give a strong contribution to $\rho_{t}$ from the acoustic plasmon pole, through both $\operatorname{Im} \chi\left(q, \omega_{p}\right)$ and $\epsilon\left(q, \omega_{p}\right)$. The optic plasmon has a higher energy, and therefore makes a smaller contribution to the integral in Eq. (2). Detailed calculations $[4,17]$ show a plasmon enhancement of the scaled transresistivity $\rho_{t} T^{-2}$ around $0.2 T_{F}$, which peaks close to $0.5 T_{F}$. For $T>0.5 T_{F}$ the strong coupling between the plasmons and the SPEs causes Landau damping of the two modes and the plasmon enhancement of the drag diminishes [4,17].

Samples A and B were fabricated from two similar wafers grown by molecular beam epitaxy, and consisted of two $200 \AA$ wide modulation-doped GaAs quantum

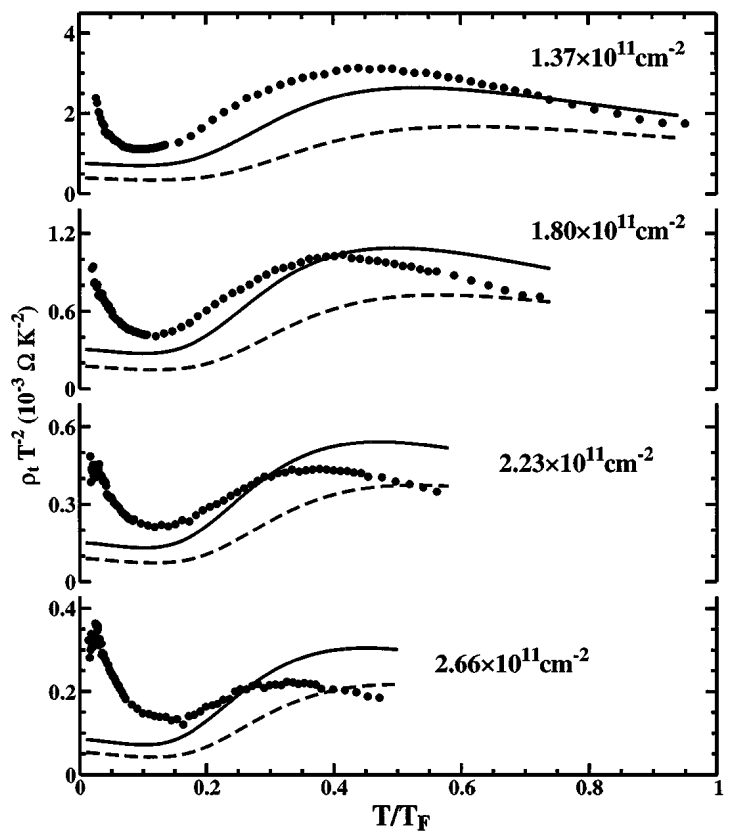

FIG. 1. The scaled transresistivity $\rho_{t} T^{-2}$ versus the reduced temperature $T / T_{F}$ of sample A, for matched carrier densities $n=1.37,1.80,2.23$, and $2.66 \times 10^{11} \mathrm{~cm}^{-2}$. The dashed (solid) lines are RPA (Hubbard) calculations of the scaled transresistivity. The Hubbard approximation with zero temperature local field corrections improves the agreement between theory and experiment in the low temperature region, but overestimates the correlations at higher temperatures. wells separated by a $300 \AA \mathrm{Al}_{0.67} \mathrm{Ga}_{0.33}$ As barrier. The resulting center-to-center separation of the two 2DEGs is $d=500 \AA$. Patterned back-gates were defined in a buried $n^{+}$GaAs layer using in situ focused ion beam lithography [18]. A Hall bar mesa $(W=67 \mu \mathrm{m}$ and $L=500 \mu \mathrm{m}$ ), NiCr:Au front-gates, and AuGeNi Ohmic contacts to the back-gates and 2DEGs were fabricated by optical lithography. Independent contacts to the two layers were then formed using a selective depletion technique $[19,20]$. Surface gates and backgates, extending over the active area of the Hall bar, were used to control independently the carrier densities of the individual layers, which were determined by four-terminal Shubnikov-de Haas and low field Hall measurements.

The zero field measurements in Figs. 1 and 2 were obtained from sample A which has an interlayer resistance $\sim 100 \mathrm{M} \Omega$. Upon application of a magnetic field the resistive voltage drop in the drive layer increases, and there is a corresponding increase in the leakage current between the layers. Sample B has an interlayer resistance greater than $1 \mathrm{G} \Omega$, and provided the magnetotransport measurements shown in Fig. 3. The zero field measurements presented for sample A were confirmed with sample B. Sample A has as-grown carrier densities of $3.3 \times 10^{11} \mathrm{~cm}^{-2}$ and $2.3 \times 10^{11} \mathrm{~cm}^{-2}$ with low temperature mobilities of $9.0 \times$ $10^{5} \mathrm{~cm}^{2} / \mathrm{V} \mathrm{s}$ and $1.3 \times 10^{5} \mathrm{~cm}^{2} / \mathrm{V} \mathrm{s}$ in the upper and lower 2DEGs, respectively. The corresponding values for sample $\mathrm{B}$ are $3.1 \times 10^{11} \mathrm{~cm}^{-2}, 2.2 \times$ $10^{11} \mathrm{~cm}^{-2}$ and $6.5 \times 10^{5} \mathrm{~cm}^{2} / \mathrm{V} \mathrm{s}, 7.6 \times 10^{5} \mathrm{~cm}^{2} / \mathrm{V} \mathrm{s}$. Samples A and B show quantitatively similar drag results.

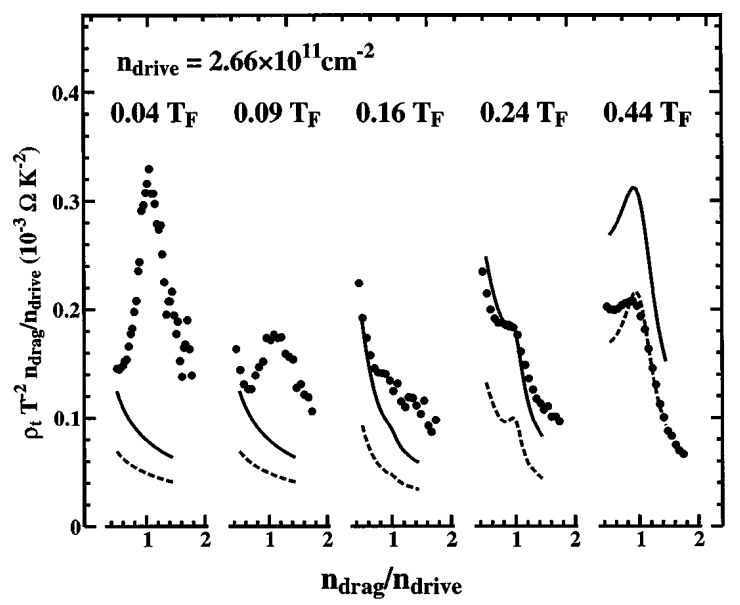

FIG. 2. The scaled transresistivity $\rho_{t} n_{\text {drag }} / n_{\text {drive }}$ of sample $\mathrm{A}$ as a function of relative carrier density $n_{\text {drag }} / n_{\text {drive }}$, for different reduced temperatures. The density of the drive layer was fixed at $n_{\text {drive }}=2.66 \times 10^{11} \mathrm{~cm}^{-2}\left(T_{F}=110 \mathrm{~K}\right)$. The dashed (solid) lines are RPA (Hubbard) calculations of the scaled transresistivity. Note that the low temperature traces are dominated by the phonon exchange mechanism and are not expected to be reproduced by theory. As in Fig. 1 we see that the Hubbard approximation overestimates the correlation effects at high temperatures, whereas the intermediate temperature regime is well reproduced by theory. 


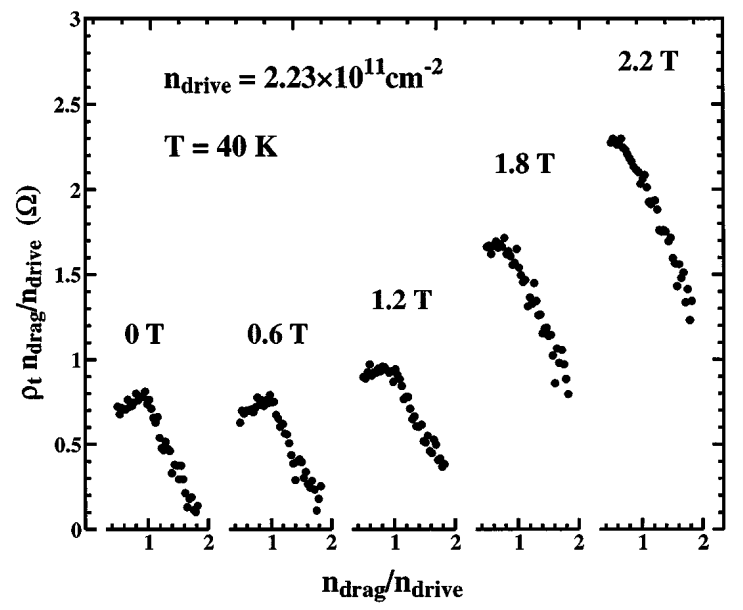

FIG. 3. The scaled transresistivity $\rho_{t} n_{\text {drag }} / n_{\text {drive }}$ of sample $\mathrm{B}$ as a function of the relative carrier density at $T=40 \mathrm{~K}$, for $B=0,0.6,1.2,1.8$, and $2.2 \mathrm{~T}$. The density of the drive layer was fixed at $n_{\text {drive }}=2.23 \times 10^{11} \mathrm{~cm}^{-2}\left(T_{F}=93 \mathrm{~K}\right)$. The figure shows how the plasmon peak disappears as the magnetoplasmons are pushed to higher energies and the coupled plasmon cannot be thermally excited.

The drag measurements were made using a circuit and ac lock-in techniques, which have been described elsewhere [6]. The measured transresistivity was identical when the roles of the upper and lower 2DEG were reversed, and $\rho_{t}$ scaled with $L$ according to Eq. (1). The drag measurements remained unchanged when the position of the earth on the drag layer was switched between the voltage probes, demonstrating that there are no interlayer leakage effects [6].

Figure 1 shows the scaled transresistivity $\rho_{t} T^{-2}$ versus $T / T_{F}$ for matched carrier densities $n=1.37$ to $2.66 \times 10^{11} \mathrm{~cm}^{-2}$. For all densities the traces show an upturn close to $0.2 T_{F}$ with a maximum close to $0.5 T_{F}$, in good qualitative agreement with theoretical predictions. The dashed lines in Fig. 1 show the scaled transresistivity based on RPA calculations [4] of the coupled plasmon dispersion relations using the carrier densities and structural parameters of our samples. These calculations evaluate the Coulomb coupling between the layers and do not include the phonon exchange that is measured below $0.1 T_{F}$.

The calculations, while reproducing the overall shape of the drag, show discrepancies with the experiment. The temperature required to excite the plasmon is lower in the experimental traces and, over most of the temperature range, the magnitude of the drag is larger than the prediction. The maximum in $\rho_{t} T^{-2}$ occurs at a lower temperature than the calculation and the decline of the enhancement at higher temperatures is more pronounced, indicating that the RPA underestimates the Landau damping effect in this regime. Both features suggest that the approximations of the RPA lead to a plasmon dispersion relation above its true value, and that a more sophisticated consideration of the collective excitations is required.
The solid lines in Fig. 1 show calculations of the scaled transresistivity, where intralayer exchange interactions are included in the Hubbard approximation. The local field calculations show qualitatively the same results as the RPA; however, the inclusion of many-body correlations lowers the plasmon energy, thereby increasing the plasmon contribution to the drag and lowering the temperature required to excite the acoustic plasmon. The Hubbard approximation provides a better fit to the data and the temperature required to excite the plasmon is better described by the theoretical curves. The position of the predicted maximum in $\rho_{t} T^{-2}$ moves to lower temperature due to the increased influence of Landau damping and the fit to the experimental data points improves. However, there is still a significant discrepancy in the magnitude of the drag. Recent theoretical work [21] has emphasized the role of many-body correlations in a Singwi-Tosi-Land-Sjölander description of the drag and gives similar results to the Hubbard approximation [22]. Both calculations use zero temperature local field corrections to describe the correlations; in contrast, the measurements are carried out at a significant fraction of the Fermi temperature, possibly leading to considerable modifications of the local fields. In fact, the zero temperature local field corrections overestimate the correlation effects at high temperatures. Hence improved finite temperature calculations are needed in order to learn more about the correlations in coupled electron gases at elevated temperatures.

Figure 2 shows the scaled transresistivity as a function of the relative carrier density, $n_{\text {drag }} / n_{\text {drive }}$, when the drive layer was fixed at $2.66 \times 10^{11} \mathrm{~cm}^{-2}$. The plasmon enhancement described by Eq. (2) depends on the product $\operatorname{Im} \chi\left(q, \omega_{p}\right)_{\text {drive }} \operatorname{Im} \chi\left(q, \omega_{p}\right)_{\text {drag }}$, which has a maximum when the boundaries of the SPE in each layer are equidistant from the acoustic plasmon dispersion curve; therefore a peak in $\rho_{t}$ is expected [4] at matched Fermi velocities, $n_{\text {drag }}=n_{\text {drive }}$. At $T=0.16 T_{F}$ the drag shows a monotonic decrease with $n_{\text {drag }} / n_{\text {drive }}$, characteristic of single-particle Coulomb scattering; the maximum at $n_{\text {drag }}=n_{\text {drive }}$ develops with increasing temperature as the SPE spectrum is smeared into the plasmon dispersion curve. The theoretical curves in the RPA and Hubbard approximation also show the evolution of the plasmon signature at matched $n$, but with the same discrepancy in magnitude that is evident in Fig. 1. When the temperature is lowered below $T=0.16 T_{F}$ the virtual phonon interaction becomes the dominant interlayer scattering mechanism and the calculated contribution from Coulomb coupling does not describe the data. Instead the experimental traces show the development of the $\rho_{t}$ maximum at matched $n$ that is characteristic of phonon exchange [10]. In summary, Fig. 2 demonstrates the crossover between the three different interlayer scattering mechanisms: phonon exchange for $T<0.1 T_{F}$, single-particle Coulomb scattering for $T \approx 0.16 T_{F}$, and plasmon enhancement for $T>0.2 T_{F}$. A further 
transition back to single-particle Coulomb scattering is expected at even higher temperatures when the plasmon modes are heavily Landau damped.

In Fig. 3 we investigate the effect of a magnetic field on the plasmon enhancement at high temperatures. The magnetic field is always such that the Landau level (LL) structure is thermally smeared and LL structure is not seen in the magnetoresistance traces of the individual layers. The scaled transresistivity is measured as a function of $n_{\text {drag }} / n_{\text {drive }}$ at $T=40 \mathrm{~K}$ for increasing $B$, when the drive layer density was fixed at $2.23 \times 10^{11} \mathrm{~cm}^{-2}$. We also observe an overall increase of $\rho_{t}$ in a magnetic field, which has previously been reported [13]. At low fields the $\rho_{t}$ maximum at $n_{\mathrm{drag}} / n_{\mathrm{drive}}=1$ has the same origin as the plasmon enhanced peak identified in Fig. 2.

In a magnetic field the magnetoplasmon dispersion relation, $\omega(B, q)=\sqrt{\omega_{c}^{2}+\omega_{p}^{2}(q)}$, is pushed to higher energies than the dispersion $\omega_{p}(q)$ at $B=0$. Therefore when $\hbar \omega_{c} \gtrsim k_{B} T$ the magnetoplasmon cannot be so easily excited and consequently the plasmon contribution decreases. Figure 3 shows the matched carrier density peak, a signature of plasmon enhancement at high temperatures, becoming weaker as $B$ is increased. The matched $n$ peak disappears at $B \approx 2 \mathrm{~T}$, the magnetic field at which $\hbar \omega_{c}$ corresponds to the $k_{B} T$ at $40 \mathrm{~K}$. At $B=2.2 \mathrm{~T}$ the transresistivity monotonically decreases with increasing $n_{\text {drag }} / n_{\text {drive }}$, displaying behavior similar to that observed at $T \approx 0.16 T_{F}$ in Fig. 2, which has been identified as single-particle Coulomb scattering. The magnetic field in Fig. 3 has an opposite effect to that of temperature in Fig. 2. By increasing the temperature at zero field we can induce a crossover from single-particle Coulomb scattering to plasmon enhancement, as the SPEs are smeared into the plasmon dispersion curves. The reverse transition can be induced with a magnetic field, lifting the magnetoplasmon energy further above the SPE boundary.

In conclusion, we have measured a plasmon enhancement of the Coulomb drag in a double quantum well system. We have observed a crossover in both temperature and magnetic field from single-particle behavior to coupled plasmon enhancement. A detailed comparison between experiment and theory shows the importance of many-body corrections to the coupled plasmon modes. The results presented in this Letter should provide a catalyst for further theoretical work into the applicability of local field corrections at elevated temperatures. The plasmon enhancement of Coulomb drag is an ideal testing ground for such research.
We thank the Engineering and Physical Sciences Research Council (U.K.) for supporting this work. J. T.N. acknowledges an EPSRC Advanced Fellowship, and D. A.R. acknowledges support from the Toshiba Cambridge Research Centre. We thank Antti-Pekka Jauho for useful discussions.

[1] D. Neilson, L. Swierkowski, J. Szymanski, and L. Liu, Phys. Rev. Lett. 71, 4035 (1993).

[2] A. Pinczuk et al., Phys. Rev. Lett. 63, 1633 (1989).

[3] J. P. Eisenstein, L. N. Pfeiffer, and K. W. West, Phys. Rev. B 50, 1760 (1994).

[4] K. Flensberg and B. Y.-K. Hu, Phys. Rev. Lett. 73, 3572 (1994).

[5] M. Jonson, J. Phys. C 9, 3055 (1976).

[6] T. J. Gramila et al., Phys. Rev. Lett. 66, 1216 (1991).

[7] A. P. Jauho and H. Smith, Phys. Rev. B 47, 4420 (1993).

[8] L. Zheng and A.H. MacDonald, Phys. Rev. B 48, 8203 (1993).

[9] K. Flensberg, B. Y.-K. Hu, A.-P. Jauho, and J. M. Kinaret, Phys. Rev. B 52, 14796 (1995).

[10] T. J. Gramila et al., Phys. Rev. B 47, 12957 (1993).

[11] M.C. Bonsager, K. Flensberg, B. Y.-K. Hu, and A.-P. Jauho, Phys. Rev. Lett. 77, 1366 (1996).

[12] M. W. Wu, H. L. Cui, and N. J.M. Horing, Mod. Phys. Lett. B 10, 279 (1996).

[13] N. P. R. Hill et al., J. Phys. Condens. Matter 8, L557 (1996).

[14] S. Das Sarma and A. Madhukar, Phys. Rev. B 23, 805 (1981).

[15] G. E. Santoro and G.F. Giuliani, Phys. Rev. B 37, 937 (1988).

[16] Recently, the two plasmon dispersion curves have been observed with Raman scattering, A.S. Bhatti et al., in Proceedings of the 23rd International Conference on the Physics of Semiconductors, edited by M. Scheffler and Zimmerman (World Scientific, Singapore, 1996), p. 1899; D. S. Kainth et al. (unpublished).

[17] K. Flensberg and B. Y.-K. Hu, Phys. Rev. B 52, 14761 (1995).

[18] E.H. Linfield, G. A.C. Jones, D. A. Ritchie, and J.H. Thompson, Semicond. Sci. Technol. 9, 415 (1993).

[19] J.P. Eisenstein, L. N. Pfeiffer, and K. W. West, Appl. Phys. Lett. 57, 2324 (1990).

[20] K. M. Brown et al., J. Vac. Sci. Technol. B 12, 1293 (1994).

[21] L. Swierkowski, J. Szymanski, and Z.W. Gortel, Phys. Rev. Lett. 74, 3245 (1995).

[22] L. Swierkowski, J. Szymanski, and Z. W. Gortel (private communication). 